\title{
Between-array normalization for 450K data
}

\author{
Jonathan A. Heiss ${ }^{1 *}$ and Hermann Brenner ${ }^{1,2}$ \\ ${ }^{1}$ Division of Clinical Epidemiology and Aging Research, German Cancer Research Center (DKFZ), Heidelberg, Germany, \\ ${ }^{2}$ German Cancer Consortium (DKTK), Heidelberg, Germany
}

OPEN ACCESS

Edited by:

Xiangqin Cui,

University of Alabama at Birmingham,

USA

Reviewed by:

Michael Wu,

The University of North Carolina at

Chapel Hill, USA

Xin Ma,

Stanford University, USA

*Correspondence:

Jonathan A. Heiss,

Division of Clinical Epidemiology and Aging Research, German Cancer

Research Center (DKFZ),

Im Neuenheimer Feld 581, 69120

Heidelberg, Germany

jonathan.heiss@dkfz-heidelberg.de

Specialty section:

This article was submitted to Epigenomics and Epigenetics, a section of the journal Frontiers in

Genetics

Received: 09 January 2015 Paper pending published: 09 February 2015

Accepted: 19 February 2015

Published: 10 March 2015

Citation:

Heiss JA and Brenner H (2015)

Between-array normalization for $450 \mathrm{~K}$

data. Front. Genet. 6:92.

doi: 10.3389/fgene.2015.00092
The Illumina Infinium HumanMethylation450 BeadChip is frequently used in epigenetic research. Besides quantile normalization there is currently no standard method to normalize the data between arrays. We describe some properties of the data generated by this platform and present a normalization method based on local regression. We compare the performance of this method with other commonly used approaches in three benchmarks (correlation between 21 pairs of technical replicates, detection of differential methylation and correlation of methylation levels for smoking-associated CpG sites with smoking behavior of 655 participants of an epidemiological study). Results indicate that the proposed method improves reproducibility, whereas some commonly used methods can have adverse effects.

Keywords: 450K, Infinium, normalization, quantile normalization, DNA methylation

\section{Introduction}

DNA methylation (DNAm) is the modification of cytosine in $\mathrm{CpG}$ dinucleotides to 5 -methylcytosine. DNAm is involved in cell differentiation, regulation of gene expression and development of cancer (Dawson and Kouzarides, 2012; Hackett and Surani, 2013). Methylation levels of specific CpG sites in blood samples have been found to reflect lifestyle factors (Lim and Song, 2012; Zhang et al., 2014) and have the potential to be used as biomarkers for early detection of cancer (Mikeska and Craig, 2014). In search of biomarkers, one is typically looking for differential methylation between two groups defined by a certain outcome. When measuring DNAm in blood samples, the sought-after changes can be small in magnitude. Therefore, obtaining precise measurements is of particular importance.

If DNAm is measured with high-density microarrays, there is often systematic bias between arrays due to a variety of variable experimental conditions such as concentrations of reagents or temperature, especially when the experiments are carried out in several batches (Lazar et al., 2013). Relevant biological signals may be masked by technical differences, also called batch effects and there are two fundamental ways to deal with them. One possibility is to consider batch effects in the statistical analysis, for instance by introducing a dummy variable for the batch in a linear model. However, batch effects may alter the data in complicated ways for which the statistical model in mind may not be adequate. It might therefore be preferable to remove these technical differences in a preprocessing step.

We have measured whole blood samples from participants of an epidemiological study with the Infinium HumanMethylation450 BeadChip (450K). In the following we describe a normalization method based on local regression, which we use to remove technical differences and to improve detection of relevant signals. We compare the performance of this method with other commonly used methods, which were previously reported to perform best (Marabita et al., 2013; Fortin et al., 2014; Wu et al., 2014), in three benchmarks using these data. 


\section{Methods}

\subsection{The Chip}

The $450 \mathrm{~K}$ chip covers approximately $485,000 \mathrm{CpG}$ sites out of the 28 million CpG sites in the human genome (Stirzaker et al., 2014). The design of this chip has been described in detail elsewhere (Bibikova et al., 2011). In the following we would like to point out some important properties of the data generated by this platform and introduce some notation.

- The chip uses single-base-extension of matched probe/target duplexes with dye-linked nucleotides and resultant fluorescence to measure abundance of unmethylated and methylated DNA molecules.

- The chip uses two different probe types, Infinium I and Infinium II (from now on abbreviated with $P_{1}$ and $P_{2}$ ). $P_{1}$ is used for $\mathrm{CpG}$ dense regions like $\mathrm{CpG}$ islands, which are mostly unmethylated, $P_{2}$ is used for $\mathrm{CpG}$ sparse regions, which are mostly methylated, so there are biological differences between the genomic loci covered by $P_{1}$ and $P_{2}$ (Eckhardt et al., 2006; Dedeurwaerder et al., 2011).

- For $P_{1}$ the abundance of methylated and unmethylated DNA molecules of the same locus is measured in the same color channel, but with different beads/probes. By contrast, for $P_{2}$ these molecules bind to the same beads, but are linked with different dyes: the unmethylated abundance is measured in the red color channel (Cy5) whereas the methylated abundance is measured in the green channel ( $\mathrm{Cy} 3)$. In the following, the intensity for methylated molecules from genome locus $i$ and sample $z$ is referred to by $M_{i z}, U_{i z}$ denotes the intensity for unmethylated molecules. $P_{1}$ consists of the subsets $P_{1 g}$ and $P_{1 r}$, the probes which are measured in the green or red color channel, respectively. Each can be further divided in the set of methylated or unmethylated signals $\left(P_{1 g}^{M}, P_{1 g}^{U}, P_{1 r}^{M}, P_{1 r}^{U}, P_{2}^{M}, P_{2}^{U}\right)$.

- One cannot compare $M_{i z}$ and $M_{i^{\prime} z}$ for $i \neq i^{\prime}$, due to the different binding affinities of the target molecules to the according probes. Additionally, one cannot compare $M_{i z}$ and $M_{i z^{\prime}}$ for $z \neq z^{\prime}$, because the concentrations of DNA and dyes vary from sample to sample. Both statements equally apply for the $U$-signals.

- Instead of comparing absolute signal intensities within and between samples one may calculate $m$-values $m_{i z}=\ln \frac{M_{i z}}{U_{i z}}$. For any given ratio of methylated to unmethylated molecules, $m_{i z}$ should not change with binding affinity or DNA concentration. Alternatively, one can compute $\beta$-values $\beta_{i z}=\frac{M_{i z}}{M_{i z}+U_{i z}}$, these fall in the range $[0,1] . m$ - and $\beta$-values can be mutually transformed $\left(\beta_{i z}=\frac{\mathrm{e}^{m_{i z}}}{\mathrm{e}^{m_{i z+1}}} ; m_{i z}=\ln \frac{\beta_{i z}}{1-\beta_{i z}}\right)$.

- Nevertheless, $m_{i z}$ may still not be comparable between probes and between samples. Besides measurement errors, the $M$ and $U$ signals contain noise resulting from binding of off-target DNA molecules. In the pool of millions of species of DNA molecules, a lot of them share some non-negligible sequence similarity leading to cross-hybridization and changed equilibria (Horne et al., 2006). Approximately $6 \%$ of the probes have cross-reactive targets and these produce many false associations (Chen et al., 2013). If the noise for probe $i$ is identically distributed between samples $z$ and $z^{\prime}$, then $m_{i z}$ and $m_{i z^{\prime}}$ are still comparable.

- For most probes there are replicates (multiple beads of the same type) on the chip. The software from the manufacturer reports summarized intensities.

- After amplification the abundance of target molecules exceeds the number of probe sequences on the chip.

\subsection{Normalization}

There are methods which focus on normalizing the $m$-values of $P_{1}$ and $P_{2}$ to each other within the arrays (Maksimovic et al., 2012; Teschendorff et al., 2013). This should be done carefully, as there are biological differences between the sites covered by the different probe designs. This step might not be necessary if subsequent analysis is done at the individual probe level. For instance, when computing $p$-values for single sites with the WilcoxonMann-Whitney test for two groups of samples, precise but not accurate values are important. Also the $t$-test is invariant to scaling and shifting of the input values. For the detection of differential methylation at locus $i$, bias does not matter if it is the same for all samples. It is important to make $m$-values comparable across samples/arrays by removing the systematic biases between samples caused by batch effects.

A simple yet effective method is the quantile normalization (QN) (Bolstad et al., 2003; Marabita et al., 2013). However, it is not clear if this method is adequate for this kind of data. In the case of gene expression analysis the use of QN is justified by the assumption that only a tiny fraction of all genes is differentially expressed, therefore making the distribution of expression levels nearly equal, whereas the global methylation level may vary. In the following we present an approach which outperforms QN and is not based on such strong assumptions.

The normalization method we describe is based on the two plots shown in Figure 1. The left panel shows the raw Cy3 intensities of all probes in $P_{1 g}$ in a pair of technical replicates, the right panel shows the same for $P_{2}$. Probes are colored according to their $m$-value in the first replicate. $P_{1}$ exhibit an intensitydependent bias between the two replicates, for $P_{2}$ the bias also depends on the methylation of the targets. This violates one assumption of QN if applied to intensities, i.e., the assumption that the order of equally methylated targets does not change between samples.

First we try to correct the intensity-dependent bias by performing local regression on the intensity values. Second, we try to correct the methylation-dependent bias for $P_{2}$ by performing a local regression on the $m$-values. Both times we use a set of "housekeeping CpG sites" and a virtual array made up of the medians over all samples as reference. The first step of the procedure was described previously (Wang et al., 2012), with the difference that we normalize both color channels separately and use only a subset of the probes to learn the loess curve. The exact steps are:

\section{Adjust $\mathrm{Cy} 3$ intensities:}

- Take a set $E$ of CpG sites which should be equally methylated in all samples $z \in Z$. 
Type I / Cy3 intensities

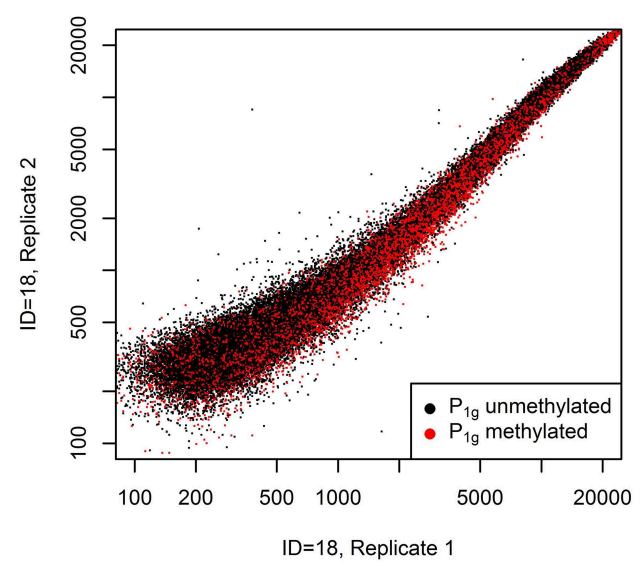

FIGURE 1 | Systematic bias between a pair of technical replicates. Raw Cy3 intensities from a pair of technical replicates from dataset $A$ (see section Benchmarks) for all probes in $P_{1 g}$ (left panel) or $P_{2}$ (right panel). Probes with a $m$-value less than zero in the first replicate are plotted in black,

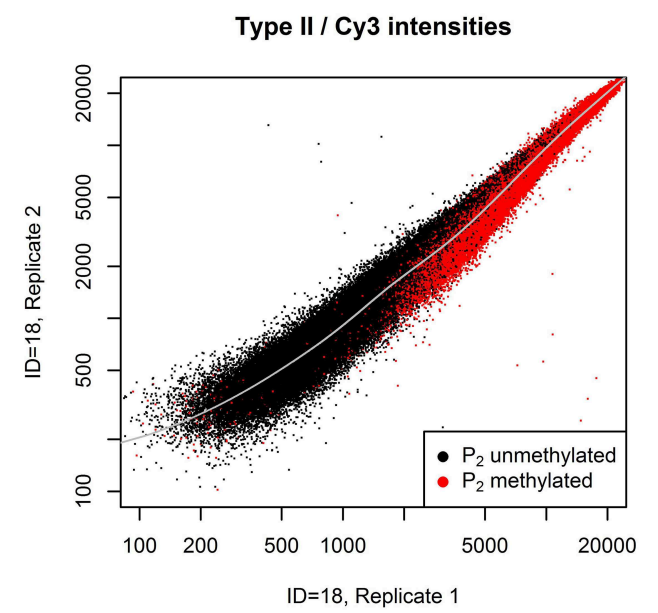

otherwise in red. Probes in $P_{1}$ show an intensity-dependent bias between the technical replicates, whereas for probes in $P_{2}$ bias also depends on the methylation of the targets: unmethylated probes are mostly above the gray line, methylated probes below.
- Compute for each probe $i \in E \cap P_{1 g}$ a reference value $S_{i *}=\operatorname{median}\left(\ln S_{i z}\right), z \in Z$. $S_{i z}$ can be an $M$ or $U$ signal. These values make up a virtual reference array which we use as baseline.

- For each sample $z \in Z$ perform a local regression with $\ln S_{i z}$ as explanatory variable and $\ln S_{i z}-\ln S_{i *}$ as dependent variable to learn the loess curve $f_{g}$.

- Compute the normalized intensities $S_{i z}^{\prime}=S_{i z} \cdot \mathrm{e}^{-f_{g}\left(\ln S_{i z}\right)}$. Normalize the Cy3 intensities of $P_{2}$ using $f_{g}$ as well. Estimating a loess curve for $P_{2}$ separately gives inferior performance, maybe because the two sources of bias (intensitydependent, methylation-dependent) are entangled.

\section{Adjust Cy5 intensities:}

- Repeat above steps for $P_{1 r}$ and the Cy5 intensities of $P_{2}$.

3. Calculate $m$-values $m_{i z}^{\prime}$ based on normalized intensities.

4. Adjust $m$-values of $P_{2}$ :

- Take a set $E$ of CpG sites which should be equally methylated in all samples $z \in Z$.

- Compute for each probe $i \in E \cap P_{2}$ a reference value $m_{i *}=\operatorname{median}\left(m_{i z}^{\prime}\right), z \in Z$.

- For each sample $z \in Z$ perform a local regression with $m_{i z}^{\prime}$ as explanatory variable and $m_{i z}^{\prime}-m_{i *}$ as dependent variable to learn the loess curve $f_{2}$.

- Compute the normalized $m$-values $m_{i z}^{\prime \prime}=m_{i z}^{\prime}-f_{2}\left(m_{i z}^{\prime}\right)$.

Carrying out the above steps we get normalized $m$-values for all probes. For the set $E$ CpG sites covering the exons of a list of housekeeping genes were used (Eisenberg and Levanon, 2013). This set is likely to contain some variable CpG sites, but local regression is robust against outliers. In total 843 probes for $P_{1 g}$, 1879 for $P_{1 r}$ and 5284 for $P_{2}$ were used. A list of these probes is provided in the supplement. In contrast to the situation for cDNA microarrays, using housekeeping CpG sites for DNAm arrays does not suffer from the fact that the expression levels of these genes do not span the entire intensity range. There are some extreme values not contained in the interval of raw signal intensities or $m$-values of the reference array, but the numbers are low. For dataset A (see below) only 12,245 measurements $(0.6 \%)$ are affected.

\subsection{Benchmarks}

To assess the performance of different normalization methods we used $450 \mathrm{~K}$ data from whole blood samples from participants of the ESTHER study (Breitling et al., 2011). In the ESTHER study patients aged between 50 and 74, who had a health check-up by their general practitioner, were recruited. Questionnaires for doctors and patients were used. These samples were combined to two datasets. Dataset A consists of 21 pairs of technical replicates distributed over 12 96-well plates, while no pair was allocated on the same plate. Dataset B consists of 655 samples (no replicates) together with age and self-reported smoking behavior (only data from current or never smokers were used) of the participants. The ESTHER study was approved by the ethics committee of the Medical Faculty of the University of Heidelberg and informed consent was obtained from all patients.

The performance of different normalization methods was evaluated in three benchmarks. The first benchmark tested if normalization increases the correlation between technical replicates of dataset A. As both measures of methylation are heteroscedastic (see Du et al., 2010 and Figure 2), we computed the correlation for $m$ - and $\beta$-values. The former highlight the performance for mostly (un)methylated sites, the latter highlight the performance for sites with intermediate methylation. Non-CpG probes and probes located on the $\mathrm{X}$ and $\mathrm{Y}$ chromosome were excluded.

The second benchmark assessed which normalization method worked best for detecting differential methylation. Dataset A was splitted in half by splitting each pair of technical replicates up. For both halves samples were grouped by sex and probes ranked 


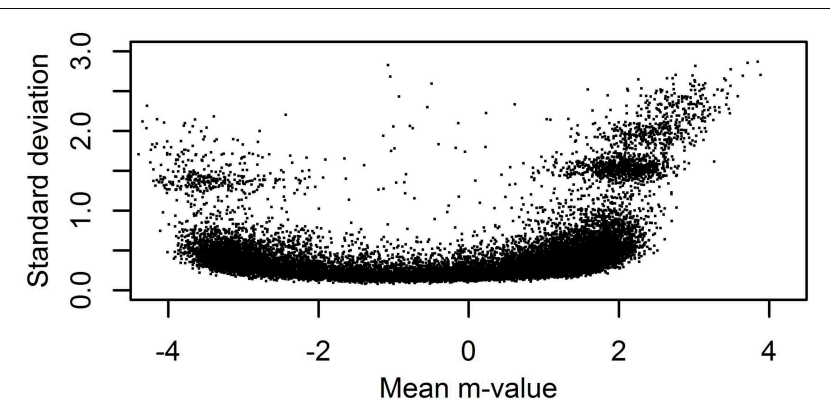

FIGURE 2 | Heteroscedasticity of $\boldsymbol{m}$-values. For a single 450K sample from the ESTHER study with unsummarized intensities the standard deviation and mean of single-bead $m$-values for probes in $P_{2}$ with at least 20 beads on the chip were computed.

according to their $p$-values from a two-sample $t$-test (using $m$ values). We plotted the concordance between the two rankings from both halves by calculating the overlap percentage of the top $k$ hits as done in Fortin et al. (2014). Again non-CpG probes and probes located on the $\mathrm{X}$ and $\mathrm{Y}$ chromosome were excluded in order to include QN in the benchmark. It does not matter for this benchmark if probes show differential methylation only due to cross-hybridization.

The third benchmark looked at known biomarkers for age and smoking. Spearman correlations of the methylation levels of three age-related CpG sites (Garagnani et al., 2012) with chronological age of the participants in dataset $\mathrm{B}$ were computed. Methylation of CpG site cg03636183 is strongly associated with current and long-term smoking exposure (Zhang et al., 2014). Spearman correlation of this biomarker with self-reported smoking behavior in dataset B was computed. Smoking exposure was assessed by three variables: smoking status with the categories never smoker $(n=469)$ or current smoker $(n=186)$, cumulative exposure (packyears) for current smokers, and the average number of cigarettes smoked per day (numcig) for current smokers. A detailed description of the variable definitions and the study population can be found in Zhang et al. (2014). For another 31 smoking-related sites [see Table 2 in Zeilinger et al. (2013)] Spearman correlations of methylation levels with smoking status were computed. Normalization should increase absolute values of the Spearman correlations.

We used .idat files for our analyses. All computations and statistical analyses were performed using R (R Core Team, 2014) and Bioconductor (Gentleman et al., 2004). R code for the first two benchmarks is provided online. As we normalized $P_{1}$ and $P_{2}$ differently, we also report the results for the first two benchmarks separately.

\section{Results}

We compared the normalization approach described above (LOESS) with the following: the standard method from the chip manufacturer without background correction (ILLU), the SWAN method (Maksimovic et al., 2012) and the FUNctional normalization (Fortin et al., 2014) as implemented in the minfi $\mathrm{R}$ package (Aryee et al., 2014). The BMIQ method (Teschendorff et al., 2013) with the $\mathrm{R}$ script version 1.3 downloaded from code.google.com/p/bmiq/We. QN applied to intensities of $P_{1 g}, P_{1 r}, P_{2}^{M}$ and $P_{2}^{U}$ separately (QN1); this way each combination of color channels and probe types is normalized separately. QN applied to raw $m$-values of $P_{1 g}, P_{1 r}$ and $P_{2}$ separately (QN2). For QN1 and QN2 only autosomal probes were normalized, as dataset A includes samples from both sexes.

Figure 3 shows the results of the first benchmark for the four combinations of probe type $\left(P_{1}, P_{2}\right)$ and methylation measure $(m, \beta)$. Two numbers on top of each boxplot and normalization method indicate how often the method achieved the highest correlation for a pair of technical replicates and how often the correlation declined compared to the raw values. As expected for ILLU and $\left(P_{1}, m\right),\left(P_{1}, \beta\right),\left(P_{2}, m\right)$ the correlations are virtually unchanged, as this method mainly scales intensities. Only for $\left(P_{2}, \beta\right)$ it increased correlations. SWAN increased correlations for $\left(P_{1}, m\right)$ and $\left(P_{2}, \beta\right)$, but had adverse effects for $\left(P_{1}, \beta\right)$ and $\left(P_{2}, m\right)$. As expected for BMIQ correlations for $\left(P_{1}, m\right)$ and $\left(P_{1}, \beta\right)$ were virtually unchanged, but it reduced the correlations for $\left(P_{2}, m\right)$ for all 21 pairs of technical replicates. QN1 performed well for $\left(P_{1}, m\right)$ and $\left(P_{1}, \beta\right)$, but produced mixed results for $\left(P_{2}, m\right),\left(P_{2}, \beta\right)$. QN2 was only favorable for $\left(P_{1}, \beta\right)$. FUN performed well for $\left(P_{1}, \beta\right)$, but produced mixed results for $\left(P_{1}, m\right),\left(P_{2}, \beta\right)$ and had adverse effects for $\left(P_{2}, m\right)$. LOESS achieved the highest correlation for all 21 pairs of technical replicates for $\left(P_{1}, m\right),\left(P_{2}, m\right)$ and had a clear lead for $\left(P_{1}, \beta\right),\left(P_{2}, \beta\right)$. It was the only method that improved correlation in every single case.

Figure 4 shows the results of the second benchmark. For the sake of clarity only the lines for NONE and the three best performing methods, LOESS, QN1 and QN2, are highlighted, and $k$ is limited to $[1,400]$, as for higher values the order of the lines did not change. QN1 and LOESS were on par for $P_{1}$, followed by QN2. QN2 performed best for $P_{2}$, followed by LOESS and QN1. Some methods had again adverse effects.

Table 1 shows the results of the third benchmark for the agerelated biomarkers and cg03636183. In all cases LOESS improved the Spearman correlation (for cg06639320 and age about 0.16) and it achieved the highest absolute value of all normalization methods in 4 out of 6 cases. The results for the other 31 smokingrelated sites are provided in the supplement. In summary, for 26 out of $31 \mathrm{CpG}$ sites LOESS improved the correlation (ILLU 26, SWAN 25, BMIQ 25, QN1 24, QN2 25, FUN 27) and it achieved the highest absolute value for $12 \mathrm{CpG}$ sites (NONE 1, ILLU 2, SWAN 3, BMIQ 1, QN1 3, QN2 9, FUN 0). 28 of the 31 sites are in $P_{2}$.

\section{Discussion}

We show that the normalization method described here reduces the differences between technical replicates more effectively than QN and improves detection of differential methylation. For known biomarkers the associations of methylation levels with the respective traits become more evident. At the same time our method does not rely on such strong assumptions like QN, and probes located on the sex chromosomes can be normalized as well. Only a small set of sites $(\sim 1.6 \%$ of all probes) equally 

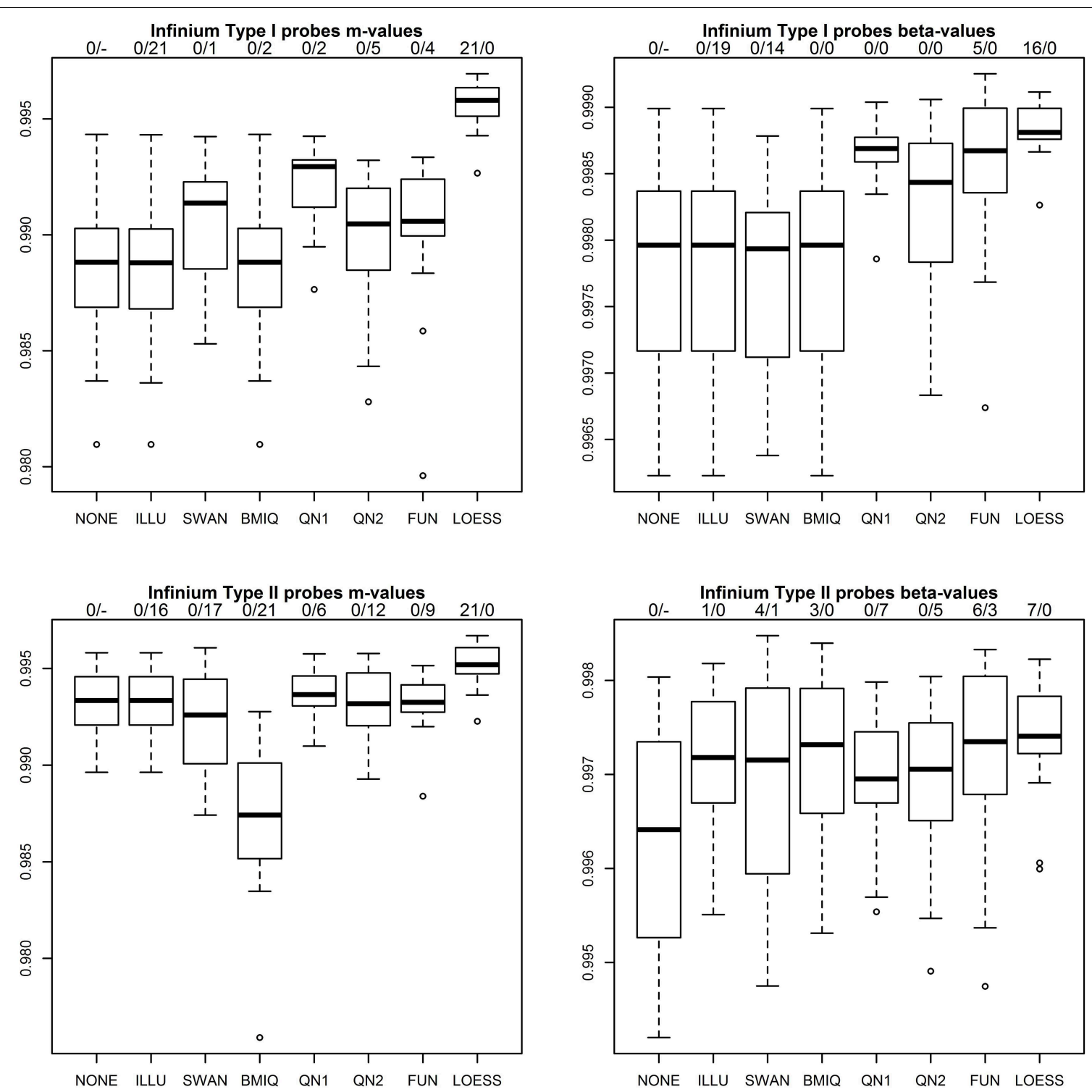

FIGURE 3 | Correlation of $\boldsymbol{m}$-values and $\beta$-values between technical replicates after various normalization methods for $\boldsymbol{P}_{\mathbf{1}}$ (top row) and $\boldsymbol{P}_{\mathbf{2}}$ (bottom row). Methods used include raw values (NONE), the standard method in the GenomeStudio software from the chip manufacturer (ILLU), the SWAN method (Maksimovic et al., 2012), the BMIQ method (Teschendorff et al., 2013), quantile normalization

methylated across all samples is required. This makes LOESS especially useful when QN cannot be applied, for example in case of global methylation changes between samples.

For the detection of differential methylation one should be careful when using within-array normalization, as in our analysis these methods had often adverse effects. Most importantly we argue that, although different probes are not comparable even if they are of the same probe type, this does not affect detection of differential methylation in a univariate screening. If there is need for accurate quantification of methylation levels, other experimental methods should be considered. We also show that even when the assumption of equal distribution of methylation levels between samples is valid, absolute intensity values of $P_{2}$ are still not comparable. The following argument might justify the use of QN1, at least for $P_{1}$. Suppose there are two samples with an identical epigenome. If one changes an arbitrary number of completely unmethylated $\mathrm{CpG}$ sites (of type $P_{1}$ ) to completely methylated or vice versa, the distribution of intensity values stays the same (high $U$ signal and low $M$ signal $\rightarrow$ low $U$ and high $M$ signal in th same color channel with the same affinity).

Most of the time the separation between low and high $\mathrm{m}$ values is not that clear as in Figure 1. We do not know the cause 


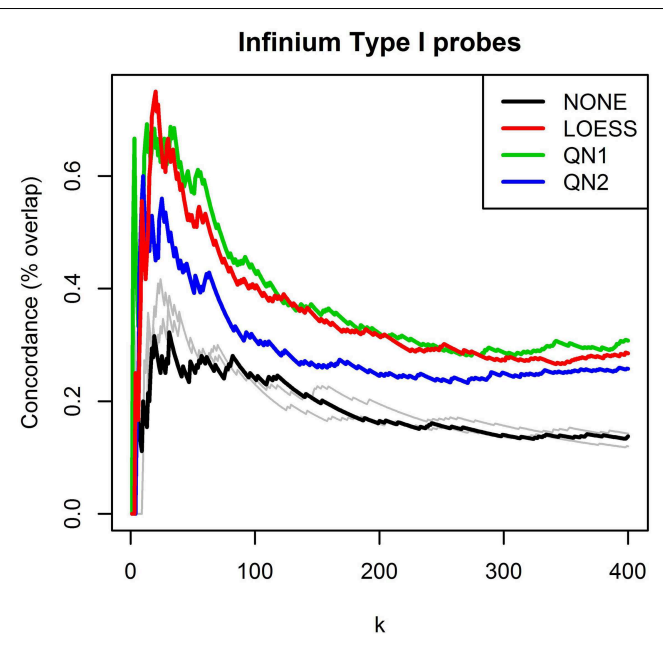

FIGURE 4 | Concordance between rankings for differential methylation. Technical replicates from dataset A were splitted to discovery and validation set and subsequently grouped by sex. Probes were ranked according to $p$-values from a two-sample

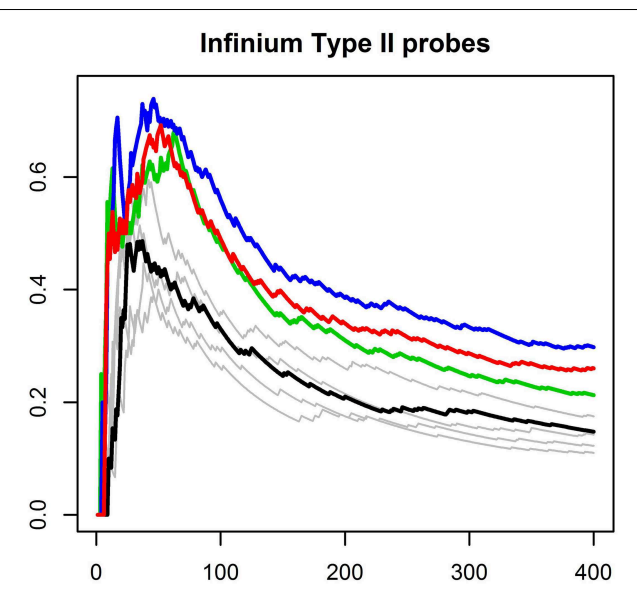

t-test. The plots show overlap percentage between the two rankings for the top $k$ hits for $P_{1}$ and $P_{2}$ separately. For clarity, only the best performing methods are highlighted, the rest are plotted in gray.

TABLE 1 | Results of the third benchmark.

\begin{tabular}{|c|c|c|c|c|c|c|c|c|c|}
\hline Trait & Biomarker & NONE & ILLU & SWAN & BMIQ & QN1 & QN2 & FUN & LOESS \\
\hline Smoking status & cg03636183 & -0.665 & -0.674 & -0.678 & -0.672 & -0.674 & -0.676 & -0.671 & -0.679 \\
\hline Packyears & cg03636183 & -0.324 & -0.355 & -0.353 & -0.335 & -0.351 & -0.328 & -0.338 & -0.346 \\
\hline Numcig & cg03636183 & -0.281 & -0.320 & -0.328 & -0.305 & -0.331 & -0.328 & -0.303 & -0.345 \\
\hline Age & cg16867657 & 0.648 & 0.648 & 0.605 & 0.648 & 0.672 & 0.648 & 0.654 & 0.682 \\
\hline Age & cg06639320 & 0.383 & 0.461 & 0.462 & 0.441 & 0.484 & 0.457 & 0.446 & 0.490 \\
\hline Age & cg16419235 & 0.441 & 0.441 & 0.431 & 0.441 & 0.468 & 0.441 & 0.441 & 0.458 \\
\hline
\end{tabular}

Spearman correlations of age or smoking behavior with methylation levels of known biomarkers.

of this pattern. It may be due to a combination of competitive effects (remember that DNA is in excess) and different binding affinities for unmethylated and methylated targets (but probes in Figure 1 do not cluster according to the number of underlying CpG sites).

Of course not all batch effects can be removed by our method. In particular we consider bias connected to signal intensities and methylation levels. However, there might be other sources of bias, like bias connected to the sequence of a probe. A good study design is still crucial.

Our results for SWAN are not in conflict with the evidence presented by Maksimovic et al. (2012), as they only looked at distributions of methylation levels, which have no significance for the conclusions they have drawn, also noted by Pidsley et al. (2013). An extensive evaluation of existing normalization methods can be found in Marabita et al. (2013) and Wu et al. (2014). The authors also tested SWAN and show that this method can reduce the correlation between technical replicates. The results for the methods ILLU and QN1 are not comparable with these papers, since we performed ILLU without background correction, because it was unfavorable, and normalized $P_{1 g}, P_{1 r}, P_{2}^{U}$ and $P_{2}^{M}$ separately for QN1. Marabita et al. recommend a combination of QN and BMIQ.
One strength of our work is the high number of samples used in the benchmarks, 21 pairs of technical replicates and 655 blood samples from a well-described study population, collected and handled in a consistent way. There are however some limitations of this work. We used correlation as a measure of similarity of replicates in the first benchmark. A distance measure, which accounts for the heteroscedasticity of the data, would be more adequate. Another issue is the set of housekeeping CpG sites. We used probes covering exons of genes showing constant expression levels across a wide range of tissues (Eisenberg and Levanon, 2013). Identifying probes with constant methylation directly would be a better approach, but this would require a dataset of samples from different tissues measured on the same $450 \mathrm{~K}$ plate, in order to minimize batch effects. Although the selected $\mathrm{CpG}$ sites should work for a wide range of tissues, we did not test this. Certainly this set needs to be refined as it will contain many sites which are not invariant. In the current implementation, measurements outside the range of values in the reference set are discarded, extrapolating would reduce the number of missing values. We also did not test how this normalization method operates with different parameter settings for the LOESS function in order to avoid overfitting. There is need for further investigations of these issues. 


\section{Funding}

The ESTHER study was funded by the Baden-Württemberg State Ministry of Science, Research and Arts.

\section{Acknowledgments}

We thank the microarray unit of the DKFZ Genomics and Proteomics Core Facility, especially Matthias Schick and Roger Fischer, for providing the Illumina Human Methylation

\section{References}

Aryee, M. J., Jaffe, A. E., Corrada-Bravo, H., Ladd-Acosta, C., Feinberg, A. P., Hansen, K. D., et al. (2014). Minfi: a flexible and comprehensive Bioconductor package for the analysis of Infinium DNA methylation microarrays. Bioinformatics 30, 1363-1369. doi: 10.1093/bioinformatics/btu049

Bibikova, M., Barnes, B., Tsan, C., Ho, V., Klotzle, B., Le, J. M., et al. (2011). High density DNA methylation array with single CpG site resolution. Genomics 98, 288-295. doi: 10.1016/j.ygeno.2011.07.007

Bolstad, B. M., Irizarry, R. A., Astrand, M., and Speed, T. P. (2003). A comparison of normalization methods for high density oligonucleotide array data based on variance and bias. Bioinformatics 19, 185-193. doi: 10.1093/bioinformatics/19.2.185

Breitling, L. P., Yang, R., Korn, B., Burwinkel, B., and Brenner, H. (2011). Tobaccosmoking-related differential DNA methylation: $27 \mathrm{~K}$ discovery and replication. Am. J. Hum. Genet. 88, 450-457. doi: 10.1016/j.ajhg.2011.03.003

Chen, Y.-A., Lemire, M., Choufani, S., Butcher, D. T., Grafodatskaya, D., Zanke, B. W., et al. (2013). Discovery of cross-reactive probes and polymorphic CpGs in the Illumina Infinium HumanMethylation450 microarray. Epigenetics 8, 203-209. doi: 10.4161/epi.23470

Dawson, M. A., and Kouzarides, T. (2012). Cancer epiGenetics: from mechanism to therapy. Cell 150, 12-27. doi: 10.1016/j.cell.2012.06.013

Dedeurwaerder, S., Defrance, M., Calonne, E., Denis, H., Sotiriou, C., and Fuks, F. (2011). Evaluation of the Infinium methylation 450K technology. Epigenomics 3, 771-784. doi: 10.2217/epi.11.105

Du, P., Zhang, X., Huang, C.-C., Jafari, N., Kibbe, W. A., Hou, L., et al. (2010). Comparison of Beta-value and $\mathrm{M}$-value methods for quantifying methylation levels by microarray analysis. BMC Bioinformatics 11:587. doi: 10.1186/14712105-11-587

Eckhardt, F., Lewin, J., Cortese, R., Rakyan, V. K., Attwood, J., Burger, M., et al. (2006). DNA methylation profiling of human chromosomes 6, 20 and 22. Nat. Genet. 38, 1378-1385. doi: 10.1038/ng1909

Eisenberg, E., and Levanon, E. Y. (2013). Human housekeeping Genes, revisited. Trends Genet. 29, 569-574. doi: 10.1016/j.tig.2013.05.010

Fortin, J. P., Labbe, A., Lemire, M., Zanke, B. W., Hudson, T. J., Fertig, E. J., et al. (2014). Functional normalization of 450k methylation array data improves replication in large cancer studies. Genome Biol. 15:503. doi: 10.1186/s13059014-0503-2

Garagnani, P., Bacalini, M. G., Pirazzini, C., Gori, D., Giuliani, C., Mari, D., et al. (2012). Methylation of ELOVL2 Gene as a new epiGenetic marker of age. Aging Cell 11, 1132-1134. doi: 10.1111/acel.12005

Gentleman, R. C., Carey, V. J., Bates, D. M., Bolstad, B., Dettling, M., Dudoit, S., et al. (2004). Bioconductor: open software development for computational biology and bioinformatics. Genome Biol. 5:R80. doi: 10.1186/gb-2004-5-10-r80

Hackett, J. A., and Surani, M. A. (2013). DNA methylation dynamics during the mammalian life cycle. Philos. Trans. R. Soc. Lond. B Biol. Sci. 368:20110328. doi: 10.1098/rstb.2011.0328

Horne, M. T., Fish, D. J., and Benight, A. S. (2006). Statistical thermodynamics and kinetics of DNA multiplex hybridization reactions. Biophys. J. 91, 4133-4153. doi: 10.1529/biophysj.106.090662 arrays and related services; Anette Albrecht-Schmitt for laboratory work; Yan Zhang for providing the data of the smoking behavior; and Katja Butterbach for helpful advice.

\section{Supplementary Material}

The Supplementary Material for this article can be found online at: http://www.frontiersin.org/journal/10.3389/fgene. 2015.00092/abstract

Lazar, C., Meganck, S., Taminau, J., Steenhoff, D., Coletta, A., Molter, C., et al. (2013). Batch effect removal methods for microarray Gene expression data integration: a survey. Brief. Bioinform. 14, 469-490. doi: 10.1093/bib/bbs037

Lim, U., and Song, M.-A. (2012). Dietary and lifestyle factors of DNA methylation. Methods Mol. Biol. 863, 359-376. doi: 10.1007/978-1-61779-612-8_23

Maksimovic, J., Gordon, L., and Oshlack, A. (2012). SWAN: subset-quantile within array normalization for Illumina Infinium HumanMethylation450 BeadChips. Genome Biol. 13:R44. doi: 10.1186/gb-2012-13-6-r44

Marabita, F., Almgren, M., Lindholm, M. E., Ruhrmann, S., Fagerström-Billai, F., Jagodic, M., et al. (2013). An evaluation of analysis pipelines for DNA methylation profiling using the illumina HumanMethylation450 BeadChip platform. Epigenetics 8, 333-346. doi: 10.4161/epi.24008

Mikeska, T., and Craig, J. M. (2014). DNA methylation biomarkers: cancer and beyond. Genes (Basel) 5, 821-864. doi: 10.3390/genes5030821

Pidsley, R., Y Wong, C. C., Volta, M., Lunnon, K., Mill, J., and Schalkwyk, L. C. (2013). A data-driven approach to preprocessing illumina $450 \mathrm{~K}$ methylation array data. BMC Genomics 14:293.

R Core Team (2014). R: A Language and Environment for Statistical Computing. Vienna: R Foundation for Statistical Computing.

Stirzaker, C., Taberlay, P. C., Statham, A. L., and Clark, S. J. (2014). Mining cancer methylomes: prospects and challenges. Trends Genet. 30, 75-84. doi: 10.1016/j.tig.2013.11.004

Teschendorff, A. E., Marabita, F., Lechner, M., Bartlett, T., Tegner, J., GomezCabrero, D., et al. (2013). A beta-mixture quantile normalization method for correcting probe design bias in Illumina Infinium $450 \mathrm{k}$ DNA methylation data. Bioinformatics 29, 189-196. doi: 10.1093/bioinformatics/bts680

Wang, D., Zhang, Y., Huang, Y., Li, P., Wang, M., Wu, R., et al. (2012). Comparison of different normalization assumptions for analyses of DNA methylation data from the cancer genome. Gene 506, 36-42. doi: 10.1016/j.gene.2012.06.075

Wu, M. C., Joubert, B. R., Kuan, P.-F., Håberg, S. E., Nystad, W., Peddada, S. D., et al. (2014). A systematic assessment of normalization approaches for the Infinium $450 \mathrm{~K}$ methylation platform. Epigenetics 9, 318-329. doi: 10.4161/epi.27119

Zeilinger, S., Kühnel, B., Klopp, N., Baurecht, H., Kleinschmidt, A., Gieger, C., et al. (2013). Tobacco smoking leads to extensive genome-wide changes in DNA methylation. PLoS ONE 8:e63812. doi: 10.1371/journal.pone.0063812

Zhang, Y., Yang, R., Burwinkel, B., Breitling, L. P., and Brenner, H. (2014). F2RL3 methylation as a biomarker of current and lifetime smoking exposures. Environ. Health Perspect. 122, 131-137. doi: 10.1289/ehp.1306937

Conflict of Interest Statement: The authors declare that the research was conducted in the absence of any commercial or financial relationships that could be construed as a potential conflict of interest.

Copyright $(9) 2015$ Heiss and Brenner. This is an open-access article distributed under the terms of the Creative Commons Attribution License (CC BY). The use, distribution or reproduction in other forums is permitted, provided the original author(s) or licensor are credited and that the original publication in this journal is cited, in accordance with accepted academic practice. No use, distribution or reproduction is permitted which does not comply with these terms. 